\title{
Reestructuración, explotaciones unifamiliares y el cultivo del olivar en Andalucía
}

\author{
M. David García Brenes*
}

\begin{abstract}
In this research, we analyse the most important transformations of family work based on the results obtained through semi-structured interviews with farmers of four villages representative of olive growing in Andalucia. This work shows how the processes of economical restructuring experienced by this crop have made the work of the family head to become of great importancein the contribution of the exploitation of the family work, and thus members of the family are pushed to look for working alternatives. We also study the way in which many small farmers survive with the income obtained from other activities and the help of the rest of themembers of the family. Wealso add a few notes about how these processes have also affected Latin America and particularly M exico.
\end{abstract}

Keywords: restructuring, family agriculture, olive oil, Andalucia, rural sociology.

\section{Resumen}

En esta investigación se analizan las transformaciones más destacadas del trabajo familiar con base en los resultados obtenidos con entrevistas semiestructuradas a los agricultores de cuatro pueblos representativos del cultivo del olivar en Andalucía. El trabajo muestra cómo los procesos de reestructuración económica que ha seguido este cultivo han provocado que el trabajo del cabeza de familia resulte fundamental en la aportación del trabajo familiar a la explotación, y que los restantes miembros de la familia busquen otras ocupaciones fuera de la explotación. Además, se estudia cómo muchos pequeños agricultores sobreviven con los ingresos obtenidos en otras actividades y con la ayuda de los demás miembros de la familia. A todo ello se añaden al gunas notas de cómo estos procesos también han afectado a América Latina, y en especial, a M éxico.

Palabras clave: reestructuración, agricultura familiar, aceite de oliva, Andalucía, sociología rural. 


\section{Introducción}

El presente análisis está centrado en un territorio concreto: Andalucía; por ello, el trabajo tiene un importante componente regional. Además, es un estudio sectorial, pues comprende el aceite de oliva, que es uno de los cultivos más importantes del proceso de especialización productiva que ha seguido la agricultura andaluza en los últimos años. En la actualidad, la producción local de aceite de oliva representa $80 \%$ de la producción española, $46 \%$ de la generada en la Unión Europea, y más de $36 \%$ de la mundial.

El objetivo general en este trabajo es analizar las repercusiones más importantes de los procesos de reestructuración productiva del aceite de oliva en Andalucía sobre la aportación del trabajo familiar a la explotación. Además, se persigue estudiar este tipo de procesos en América Latina, y en particular en $\mathrm{M}$ éxico. Se intenta encontrar analogías de cómo los procesos de reestructuración productiva han afectado a estos territorios.

Además de ese objetivo general existen una serie de objetivos específicos que lo complementan. Uno de ellos es el estudio de la distribución del trabajo familiar en la explotación, analizándose la importancia que tiene el trabajo del cabeza de familia, de los hijos y el de la mujer. Esto nos permitirá conocer la implicación de cada uno de los miembros de la familia en la explotación, la importancia del relevo generacional y el papel que tiene la mujer en este cultivo. También se estudia la reproducción social de estas explotaciones y su permanencia en el futuro.

Para llevar a cabo los objetivos anteriores, en esta investigación se cuenta con una serie de aportaciones teóricas. Entre ellas destaca la recogida en la obra "Ecosociología: algunos elementos teóricos para el análisis de la coevolución social y ecológica en la agricultura", de Eduardo Sevilla y M anuel G onzález (1990), que estudian las estrategias que mantienen las unidades domésticas como respuestas de resistencia o adaptación, de acuerdo con su lógica reproductiva. Para el caso de M éxico se cuenta, entre otros, con el trabajo "América Latina: descomposición y persistencia de lo campesino", de Víctor M anuel Figueroa (2005).

También hay que destacar algunos trabajos que toman como base la economía ecológica, pero que se refieren de un modo particular al cultivo del olivar; entre ellos, "La crisis del olivar como cultivo biológico tradicional", de José M anuel Naredo (1983), que ha apoyado el estudio de los procesos de 
reestructuración y el tránsito del olivar tradicional a un cultivo moderno.

Por último cabe señalar que este trabajo también recibe las aportaciones de la sociología cualitativa; destaca en esta área el artículo "Perspectivas de la investigación social: el diseño en las tres perspectivas", de J esús I báñez (2002). De este modo, se apoya el trabajo en los resultados obtenidos con una serie de entrevistas semiestructuradas a los propios agricultores, quienes son los principales actores y informantes privilegiados para explicar la realidad.

\section{Características básicas del olivar andaluz}

El olivar es un cultivo esencialmente mediterráneo. Prueba de ello es que en esa zona se encuentra $98 \%$ de la superficie mundial cultivada. ${ }^{1}$ De un modo particular, es un cultivo especialmente importante en la rivera norte, en los países considerados como "mediterráneos" integrados en la Unión Europea (España, Grecia, Italia y Portugal), donde en 2001 se concentró 75.5\% de la producción mundial (Consejo O leícola Internacional, 2002).

El olivar andaluz ha tenido a lo largo de la historia un destacado papel en el contexto español, por su participación tanto en la superficie como en la producción total. Este importante papel se ha acentuado con el paso del tiempo. Así, la participación andaluza en cuanto a superficie cultivada en el conjunto del Estado Español en el periodo de 1943 a 2001 ha pasado del 49.7 a $60.1 \%$ (véase cuadro 1). Respecto a la producción, en el periodo de 1962 a 2001 la participación andaluza pasó de 69.5 a $80.7 \%$ (véase cuadro 2).

D entro de España, a Andalucía le siguen a gran distancia C astilla-La M ancha y Extremadura. Así, en 2001 estas regiones suponían 15.5 y $10 \%$ de la superficie y 6.4 y $4.4 \%$ de la producción total, respectivamente. Esta creciente importancia del olivar andaluz se relaciona con la evolución de su productividad. Así, si a principios de la década de los sesenta la productividad en Andalucía de la hectárea cultivada era por término medio de $196.3 \mathrm{~kg}$ de aceite/ha, en 2001 fue de $560.5 \mathrm{~kg} / \mathrm{ha}$. M ientras tanto, el olivar de la región que ocupa el segundo lugar en el ranking español

${ }^{1}$ De ello, $90 \%$ corresponde al olivar destinado a la producción de aceite de oliva, y el $10 \%$ restante, a la producción de aceituna de mesa como alimento. En el primer caso, de cada cien kilogramos de aceitunas se obtienen como media veinte kilogramos de aceite. 
C uadro 1
Superficie de olivar (\% sobre la total)

\begin{tabular}{lrrrc}
\hline C. Autónoma & 1943 & 1963 & 1983 & 2001 \\
\hline Andalucía & 49.7 & 52.3 & 57.8 & 60.1 \\
Castilla-La M ancha & 14.7 & 14.8 & 13.8 & 15.5 \\
Extremadura & 9.2 & 9.7 & 12.0 & 10.0 \\
Cataluña & 10.0 & 9.3 & 6.1 & 5.2 \\
Resto* & 16.4 & 13.9 & 10.3 & 9.2 \\
Total & 100 & 100 & 100 & 100 \\
\hline
\end{tabular}

* Incluye Valencia, Aragón, M urcia, M adrid, Baleares, Castilla y León, N avarra, La Rioja y el País Vasco.

Fuente: Civantos (1997) para 1943, 1963 у 1983, у м APA (2001) para 2001.

\section{C uadro 2}

Producción media de aceite (\% sobre el total de toneladas)

\begin{tabular}{lccc}
\hline C. Autónoma & $1962 / 66$ & $1982 / 86$ & 2001 \\
\hline Andalucía & 69.5 & 78.1 & 80.7 \\
Castilla-La M ancha & 9.2 & 8.7 & 6.4 \\
Extremadura & 5.4 & 4.9 & 4.4 \\
Cataluña & 6.9 & 3.5 & 3.7 \\
Resto* & 9.0 & 4.8 & 4.8 \\
Total & 100 & 100 & 100 \\
\hline
\end{tabular}

*Incluye Valencia, Aragón, M urcia, M adrid, Baleares, Castilla y León, N avarra, La Rioja y el País Vasco.

Fuente: Civantos (1997) para 1943, 1963 y 1983, y м APA (2001) para 2001.

de las regiones productoras, Castilla-La M ancha, ha pasado de los 91.7 a los $172.4 \mathrm{~kg}$ de aceite/ha. Por tanto, si a principios de los sesenta la productividad andaluza era $114.1 \%$ superior a la casteIlano-manchega, en 2001 fue superior en 225.1\%. Es decir, en el periodo 1962-2001 el olivar andaluz acentuó su superioridad en términos de productividad en relación con el olivar castellanomanchego en particular, y con el español en general.

Todo ello se asocia con el proceso de especialización productiva que ha seguido la economía andaluza desde 1970. La agricultura andaluza, con una orientación cada vez más marcada hacia los mercados exteriores, se ha especializado en aquellas producciones que tienen mayor aceptación y capacidad competitiva. Así, en 1977 los sectores del olivar y de frutas y hortalizas 
acaparaban $51.8 \%$ de la producción final agraria, mientras que en 1999 esos mismos sectores suponían 80\%, donde al aceite de oliva le corresponde 30\% (Delgado y Vázquez, 2002).

Junto a la fuerte especialización productiva de la economía andaluza, otro elemento muy importante es la dualidad pequeña-gran explotación. En Andalucía existe, para un conjunto de actividades agrarias en general, y para el olivar en especial, una acusada polarización de la distribución de la propiedad de la tierra que tiene orígenes ancestrales. Esto constituye un rasgo diferenciador de la economía andaluza respecto a la gran mayoría de las regiones españolas. Eduard M alefakis (2001) divide, de hecho, con base en el régimen de la propiedad agraria, el territorio español en dos grandes zonas: la parte occidental en Andalucía y Extremadura, donde predominan las grandes propiedades y los grandes propietarios, y el resto del país, donde la actividad agrícola descansa principalmente en los pequeños propietarios que cultivan fincas de reducida extensión.

La estructura agraria de un cultivo tan emblemático para la región como es el olivar refleja, como no podía ser de otro modo, esa desigual distribución de la tierra. Sin embargo, en los últimos años se asiste a un fortalecimiento de las estructuras de la pequeña explotación frente a la gran explotación. Por ejemplo, en 1972 las explotaciones con menos de cinco hectáreas representaban $60.4 \%$ del total de explotaciones, y en 1998 esa participación había aumentado a 79.3\%. I gualmente, a esas explotaciones les correspondía 14\% de la superficie en 1972, y $26.5 \%$ en $1998 .^{2}$ Las explotaciones que tenían más de 100 hectáreas, por el contrario, han pasado de representar $2.7 \%$ en 1972 a $0.4 \%$ en 1998 , y de ocupar $31.8 \%$ de la superficie en 1972 a $13.3 \%$ en 1998. De este modo, las explotaciones más pequeñas aumentan en $18.9 \%$ su participación en el número total de explotaciones, y $12.5 \%$ en la superficie total, mientras que la participación de las explotaciones de mayor tamaño descienden 2.3 y $18.5 \%$, respectivamente (véase cuadro 3).

Así, en el periodo comprendido entre 1972 y 1998 se ha incorporado al cultivo del olivar en Andalucía un numeroso grupo de explotaciones, que en la mayoría de los casos son de pequeño tamaño y que en buena medida son el producto de la reconver-

\footnotetext{
${ }^{2}$ Estos valores se han calculado a partir de una definición muy estricta de minifundio, considerándose como tales únicamente las explotaciones con menos de cinco hectáreas. En el resto del texto se ha utilizado otra definición más amplia que considera como minifundio cualquier explotación con menos de 15 hectáreas.
} 


\section{C uadro 3 \\ Estructura de las explotaciones de olivar en Andalucía (\% sobre el total)}

\begin{tabular}{lrcrcrc}
\hline Tamaño (ha) & \multicolumn{2}{c}{1972} & \multicolumn{2}{c}{1998} & \multicolumn{2}{c}{ 1998-1972 } \\
& N úm. Superficie & \multicolumn{2}{c}{ N úm. Superficie } & N úm. Superficie \\
\hline $0-5$ & 60.4 & 14.0 & 79.3 & 26.5 & +18.9 & +12.5 \\
$5-20$ & 26.5 & 22.4 & 16.5 & 30.7 & -10.0 & +8.3 \\
$20-50$ & 7.5 & 17.8 & 2.9 & 17.8 & -4.6 & 0.0 \\
$50-100$ & 2.9 & 14.0 & 0.9 & 11.7 & -2.0 & -2.3 \\
M ás de 100 & 2.7 & 31.8 & 0.4 & 13.3 & -2.3 & -18.5 \\
Total & 100 & 100 & 100 & 100 & & \\
\hline
\end{tabular}

Fuente: INE (1972) y Consejería de Agricultura y Pesca (2000).

sión de explotaciones dedicadas anteriormente a otros cultivos como los cereales y el girasol. Todo ello ha supuesto que el cultivo del olivar haya aumentado su importancia económica y social, pues existen más de 257 mil explotaciones, y más de 240 mil son de pequeño tamaño.

\section{La reestructuración del olivar andaluz}

El olivar tradicional constituía un sistema autónomo y equilibrado. La agricultura y la ganadería eran actividades complementarias que permitían la reposición natural del suelo por las pérdidas de energía ocasionadas con el proceso productivo, sin necesidad de ninguna aportación exterior ( $\mathrm{N}$ aredo, 1996).

Además, el olivar tradicional compartía el uso del suelo con el cultivo de leguminosas, que servían para enriquecer la tierra con nitrógeno. También se dedicaban algunas tierras a pastos 0 incluso a otros cultivos como la vid. Es decir, se utilizaba la rotación de cultivos como forma de generación de abonos y fertilizantes orgánicos, de modo que la actividad agraria no precisase de fuentes energéticas externas. La explotación agraria se fundamentaba así en la gestión de un flujo cerrado de energía y materiales en el que estaban integrados los distintos espacios y usos agrarios del suelo.

Las numerosas especies de animales que vivían en el entorno de este cultivo controlaban la aparición de plagas y enfermedades. Un agricultor mantenía: "Antes en el olivar no se conocían enfermedades, las hormigas, los jilgueros, los zorzales acababan con las 
enfermedades, no faltaban ni hormigueros, ni pájaros en cualquier olivo al que te acercaras". A ello se le unían una serie de prácticas muy habituales en este cultivo, como la eliminación de la leña muerta y el encalado durante el verano, que impedían la proliferación de hongos y otros parásitos en el mismo (Parra, 1988).

La reproducción del olivar tradicional estaba controlada por los propios agricultores. La difusión del cultivo se realizaba a partir de los restos obtenidos con la poda del olivo, ${ }^{3}$ y con injertos. ${ }^{4} Y$ las variedades se elegían con base en la adaptación al sueIo, resistencia a las enfermedades, agentes atmosféricos existentes en un determinado territorio, etc. Las variedades, por tanto, no se elegían sobre la base de un simple criterio de aumento de la productividad. Esto hizo que existiera una amplia gama de variedades. Por ejemplo, en la provincia de Sevilla había 32 variedades distintas (Fernández, 1927). Además, el olivar tradicional andaluz era un cultivo que se mostraba poco exigente con el uso del suelo. En la mayoría de las ocasiones existían 50 o 60 olivos por hectárea, con una distancia de 11 o 12 metros entre ellos, y con un rendimiento medio de $1,500 \mathrm{~kg}$ de aceituna por hectárea (García, 2004).

Una vez analizadas las principales características del olivar tradicional andaluz, que lo convertían en un cultivo sostenible y ecológico, pasaremos a estudiar su carácter social. La organización del trabajo del olivar tradicional se dividía en una serie de tareas entre las que destacaba la recolección de la aceituna, porque empleaba un importante número de trabajadores. Un agricultor describía el reparto del trabajo en ésta del siguiente modo: "Los hombres se dedicaban a emparejar los suelos, colocar los telones y derribar las aceitunas con los apuraores (varas de madera), y las mujeres y los niños a recoger y limpiar las aceitunas del suelo y las derribadas". Esto hacía que fuese un trabajo completamente manual y, por tanto, con una baja productividad ( $G$ arcía, 2004).

Además, los sistemas agrarios tradicionales andaluces, en general, se basaban en la contratación de abundante mano de obra

${ }^{3}$ Con esta tarea, el agricultor consigue alcanzar el equilibrio de las funciones vegetativas y reproductivas del olivo, haciendo compatibles la máxima producción y la vitalidad del olivo (García et al., 1997).

${ }^{4}$ Esta técnica de multiplicación indirecta consiste en adherir entre la parte leñosa y la corteza de una rama cortada horizontalmente de un olivo, una yema del olivo que se pretende propagar. Los injertos se realizaban, la mayoría de las veces, sobre olivos silvestres (acebuches), pues son árboles muy vigorosos y resistentes al frío (Guerrero, 1997). 
con niveles salariales muy bajos, lo que constituía la base de la rentabilidad monetaria de esas explotaciones. Así, no se trataba de sistemas productivos orientados a la acumulación de capital mediante, por ejemplo, la compra de maquinaria para conseguir el aumento de la productividad del trabajo. N o sería sino hasta la década de los cincuenta cuando se produciría la mecanización del proceso productivo (Bernal, 1979).

El olivar tradicional andaluz era entonces un cultivo sostenible ecológicamente, creador de empleo y rentable, pero que comprendía unas condiciones de trabajo muy duras.

Sin embargo, a finales de la década de los cincuenta los pilares sobre los que se apoyaban los sistemas agrarios tradicionales andaluces experimentaron importantes transformaciones. Se asistió a un fuerte encarecimiento del coste por unidad de trabajo empleada. M anuel Delgado y Andrés Vázquez (2002) calculan que entre 1956-1960 el volumen total de salarios tuvo, para el caso de la agricultura andaluza, una tasa de crecimiento anual de $6.7 \%$. Además, se produjeron importantes transformaciones en el marco institucional que regulaba el mercado de trabajo que supusieron una reducción de la jornada laboral y el establecimiento de otras medidas con la finalidad de mejorar sustancialmente las condiciones de trabajo, como fue el caso del salario mínimo interprofesional. H ubo, por tanto, un fuerte encarecimiento del coste por unidad de trabajo empleada. Como consecuencia de ello se pondría en marcha un importante proceso de mecanización de aquellas tareas agrícolas que mayor número de trabajadores empleaban para abaratar sus costes.

En el caso del olivar, se produjo el aumento de los salarios de la recolección de la aceituna, cuyo coste representaba más de $70 \%$ de los costes totales de ese cultivo. En esas condiciones, lo lógico era esperar un proceso de sustitución del trabajo por capital. Sin embargo, distintos problemas técnicos y la propia dinámica de la actividad, en la que no se habían dado en el pasado procesos de acumulación de capital intensos, hicieron que ese proceso de mecanización finalmente no ocurriera. En efecto, el olivar tradicional era un olivar de troncos muy gruesos, con variedades como la hojiblanca (ocupaba $22.8 \%$ de la superficie) y la picuda (7.8\%), en las que la aceituna no se desprendía. Esto hacía que el empleo de los vibradores no resultara eficiente. Además, el olivar tradicional era un cultivo con una densidad muy reducida y muy longevo. Así, $80 \%$ de las explotaciones tenían menos de 100 olivos/ ha, $41.2 \%$ de los olivos censados tenían más de 100 años, y más 
de $75 \%$ de la superficie cultivada tenía rendimientos inferiores a los 1,500 kilogramos/ha (O rtega,1975). La baja densidad y la existencia de olivos viejos (por tanto más gruesos) dificultaban también la utilización de vibradores, de modo que la mecanización de la tarea de la recolección (la más importante en términos relativos) resultaba muy difícil. Por tanto, los rasgos distintivos del olivar tradicional y el nivel tecnológico que existía en aquellos momentos no permitieron la mecanización de la recolección de la aceituna.

Además, al cultivo del olivar se incorporarían los nuevos inputs para intensificar de un modo importante los niveles de producción.

\begin{abstract}
El cultivo intensivo moderno busca maximizar su producción supliendo las limitaciones del entorno físico con la inyección externa de nutrientes, agua o productos fitosanitarios, haciendo de él un sistema inestable, altamente dependiente de la aplicación de medios químicos derivados de los combustibles fósiles o de otras materias renovables. Es decir, que el cultivo intensivo moderno rompe por completo esa imagen tradicional de olivar de árbol de secano, característico por su rusticidad, menos exigente en suelo y humedad que los cultivos anuales más corrientes, para convertirlo en un cultivo distinto, mucho más exigente en agua y medios químicos ( $\mathrm{N}$ aredo, 1983: 241).
\end{abstract}

De este modo, en el cultivo del olivar andaluz se aplicarán todos los componentes de la "revolución verde"; esto es, utilización de abonos químicos, mecanización, selección de nuevas razas y variedades más productivas, etcétera.

Todos esos cambios contaron con el apoyo del Estado, que trataba de aumentar los niveles de producción de un alimento como el aceite de oliva, que tiene una enorme importancia en el presupuesto familiar español, para bajar su nivel de precios y liberalizar una parte de la renta familiar que se destinaría a la demanda de otros productos fundamentalmente industriales. Además, se produjo la aparición en el mercado español de los aceites de semillas (soja, girasol, etc.), comparativamente mucho más baratos. ${ }^{5}$ Simultáneamente, las autoridades ejercieron un control que no permitía un aumento 'libre' de los precios. La prioridad de la intervención pública en esos momentos fue el control de los precios y la garantía de los abastecimientos (López, 1980; Abad y N aredo, 1997).

${ }^{5}$ En 1953 se firmaría un acuerdo con Estados Unidos, por el que se permitía la entrada en el mercado español de una importante cantidad de aceite de soja a bajo precio (H umanes, 1996). 
En el olivar, la subida de los salarios y la incorporación de los nuevos nutrientes químicos significarían un importante aumento de los costes que no sería compensado por los incrementos de la productividad. De este modo, el proceso de modernización que seguiría el olivar andaluz en la década de los sesenta erosionaría seriamente sus niveles de rentabilidad económica.

Esa crisis originó que el Estado español pusiera en marcha, en la década de los setenta y posteriormente, en la primera mitad de los años ochenta, un proceso de reestructuración del olivar para recuperar los niveles de rentabilidad. Se favorecería la mecanización de las explotaciones y el aumento de la productividad, así como la reconversión de las explotaciones menos productivas y la desaparición de las menos competitivas. En el periodo 19721986 se arrancaron 340,000 hectáreas de la superficie de olivar en España ${ }^{6}$ (M APA, 1988).

Además, con las nuevas plantaciones ocurrió un hecho muy singular desde una perspectiva territorial. La participación de las provincias occidentales, en términos de superficie, pasó de 38.4 a $27.3 \%$, y la de las provincias orientales, de 61.6 a $72.7 \%$. El olivar más productivo se localizaba ahora en las provincias orientales, fortaleciendo el perfil de especialización agraria de las mismas (Angles, 1999). Asimismo, entre 1972 y 1986 la producción de aceite de la provincia occidental más importante (Sevilla) descendió de 36,000 a 29,000 toneladas, mientras que la de la provincia oriental más importante (J aén) pasó de 140,000 a 206,000 toneladas (Civantos, 1997). Se asistió así a un proceso de especialización del olivar en aquellos suelos con mejores cualidades para el desarrollo de ese cultivo, experimentándose una fuerte reducción en aquellas zonas en las que era menos competitivo.

La aplicación al olivar de la Política Agraria Comunitaria (PAC), tras la incorporación de la agricultura andaluza a la Comunidad Europea en 1986, provocaría la segunda reestructuración del cultivo del olivar. De nuevo, la intervención pública fomentaría la productividad a partir del sistema de precios públicos de intervención, y de un sistema de ayudas directas a la producción que diferenciaba entre pequeños productores de aceite de oliva (aquellos con una producción anual menor de 500 kilogramos de aceite) y el resto de productores. Este sistema de ayudas generó ma-

${ }^{6}$ La falta de información nos obliga a referir nuestro análisis al olivar español. Sin embargo, éste refleja en gran medida las transformaciones que haya sufrido el olivar andaluz, pues ocupa este último $60 \%$ de la superficie total. 
yores niveles de producción, para obtener más ayudas y vender más aceite, en el peor de los casos, a los precios públicos de intervención. Además, las autoridades comunitarias, conscientes de que el aumento de la productividad no garantizaba niveles de rentas aceptables para los pequeños productores, establecieron para ellos un régimen de ayudas para mejorar su situación.

Este sistema, vigente hasta bien entrados los noventa, era, por lo tanto, muy similar al que había aplicado la Administración española con anterioridad. Se fomentaba la productividad a partir del sistema de precios de intervención y de un régimen de ayudas directas a la producción. La diferencia esencial estribaba en que no se impulsaba ahora el abandono de las tierras menos productivas. Además, con la aplicación de esta política se pretendía solucionar los problemas de abastecimiento de productos agrícolas que habían aparecido tras la finalización de la Segunda Guerra M undial; aumentar la producción agrícola y bajar los precios, indispensables para la implantación del modelo de consumo de alimentos a bajo precio.

Los problemas presupuestarios ocasionados por los excedentes agrarios y las presiones de la Ronda de Uruguay, favorables a facilitar el acceso de producciones foráneas a los mercados locales, fueron argumentos suficientes para forzar una reforma de la PAC. Las reformas en la PAC sucedieron poco a poco a lo largo de la década de los noventa. La principal reforma de la política comunitaria del aceite de oliva, la más completa y la que cambia definitivamente el sentido de la intervención pública en el olivar, es la de 1998. El nuevo sistema de ayudas a la producción establece que todos los agricultores recibirán las ayudas en función exclusivamente de su producción sin diferenciar entre pequeños y grandes productores. Además, se elimina el M ecanismo de Intervención, y con ello los precios de garantía, porque cuando los precios de mercado del aceite de oliva son muy bajos, no tienen los productores la posibilidad de vender su aceite al Estado y están obligados a venderlo en las condiciones que establece el mercado.

Lo anterior significó la implantación de una política asistencial que favorece al olivar más productivo y delega al mercado la dirección y gestión de la dinámica de acumulación de ese cultivo. Así, se responde a las exigencias del nuevo modelo de acumulación que se apoya en un creciente proceso de mundialización de los mercados agrarios y en una fuerte desregulación tendente a aumentar la competencia (Cano, 2000). 
Como resultado de la aplicación de la política comunitaria han aumentado tanto la superficie como la producción de un modo muy significativo. En 1986-1992 existían en Andalucía, como media, 1'225,100 hectáreas, y en 1999-2001 se contabilizaron 1'421,300 hectáreas. Se ha producido, por tanto, un aumento de 196,200 hectáreas, lo que ha roto la tendencia a la caída de la superficie cultivada de olivar observable desde principios de los setenta (cuadro 4).

\section{C uadro 4 \\ O livar en Andalucía}

\begin{tabular}{lcc}
\hline Periodo & Superficie $\left(10^{3}\right.$ de ha) & Producción $\left(10^{3} \mathrm{tm}\right.$ de aceite $)$ \\
\hline $1986-1992$ & $1,225.1$ & 462.0 \\
$1993-1998$ & $1,304.6$ & 564.0 \\
$1999-2001$ & $1,421.3$ & 615.9 \\
\hline
\end{tabular}

Fuente: MAPA (2001) y Civantos (1997).

Pero donde se advierte un aumento más importante es en los niveles de productividad. En 1986-1992, la productividad media por hectárea era de $377.1 \mathrm{tm} / \mathrm{ha}$, y en el periodo 1999-2001 aumentó hasta 433.3, lo que supone un incremento de más de $14 \%$.

Sin embargo, al aumento de la productividad del olivar lo acompaña el crecimiento de los costes ecológicos y sociales, pues su modo de producción utiliza un número reducido de variedades, por lo que se disminuye la biodiversidad que tenía el olivar tradicional. Así, en el periodo comprendido entre 1981 y 1998 la superficie de la variedad picual (por su fácil mecanización y elevados rendimientos en aceite) pasó de representar 45.1 a $58.2 \%$ en la superficie total de olivar en Andalucía, y en el caso de la manzaniIla sevillana (por el sistema de ayudas que tiene en la Unión Europea) el crecimiento ha sido más moderado: de 4.3 a 5.0\%. El resto de las variedades, por el contrario, ha descendido enormemente, y de manera muy especial la "nevadillo blanco" y la "lechín", con una caída de 80.0 y $67.5 \%$, respectivamente. Esto ha supuesto que más de $40 \%$ la producción total de aceite de oliva en Andalucía sea generada con la variedad de aceituna picual (García, 2004).

El agua es otro de los inputs, junto con los agroquímicos, que los agricultores andaluces utilizan para aumentar la producción del olivar. Así, la superficie de olivar en regadío en Andalucía se ha multiplicado por cuatro desde 1980, pasando de 80,000 a más 
de 323,000 hectáreas (D onaire, 2005). Sin embargo, este sistema productivo que se apoya en el agua y el uso de nutrientes químicos daña el patrimonio natural. En este mismo se sentido se expresaban algunos estudios para el caso de América Latina. Así, en El Saúz, en el occidente de M éxico, el uso de agroquímicos, la intensificación del cultivo en la misma parcela y la desaparición del policultivo están disminuyendo las precipitaciones y la fertilidad del suelo, y haciendo desaparecer los conocimientos empíricos (Gerritsen et al., 2003).

Por último cabe señalar que en los últimos años ha adquirido un papel destacado el olivar ecológico. Representa $36 \%$ de la superficie en régimen de agricultura ecológica en Andalucía. La provincia de Córdoba tiene un destacado protagonismo en el desarrollo de ese sistema productivo. Así, en 2002 representó más de $50 \%$ de la superficie del olivar ecológico en Andalucía (Consejería de Agricultura y Pesca, 2005).

Se trata de una iniciativa que está en consonancia con el cambio de filosofía de la política agraria comunitaria en 1992, en favor de la protección y conservación del ambiente sobre la base de la no utilización de productos contaminantes. La Unión Europea ha establecido un sistema de ayuda por hectárea para los agricultores que mantengan un sistema agrícola ecológico. En la mayoría de los casos se trata de pequeñas y medianas explotaciones que encuentran en la producción del aceite ecológico una alternativa viable para mantener su forma de vida y como defensa frente a las estrategias comerciales de los grandes grupos alimentarios.

Además, la combinación de un producto de extraordinaria calidad como es el aceite ecológico con otros elementos como el turismo rural, se convierte en una apuesta muy importante para el aprovechamiento de los recursos locales y la dinamización de la zona en la que se desarrolla esta actividad agraria. Así, se conjuga la realización de una serie prácticas agrícolas que en buena medida protegen el ambiente, con la puesta en valor de un territorio como atractivo turístico, para favorecer el desarrollo rural de una zona que ha sido gravemente perjudicada por los procesos de globalización económica y de liberalización de los mercados.

\section{Elementos metodológicos de la investigación}

El olivar constituye un sistema productivo muy diverso. Para estudiar el trabajo familiar en el proceso de producción de ese cultivo se ha elaborado una tipología de explotaciones con base en 
la consulta a expertos y en la realización de entrevistas en profundidad a los propios agricultores. De esta forma, se han considerado básicamente dos criterios: el tamaño de las explotaciones y el rendimiento del olivar.

De acuerdo con el tamaño, se distinguen tres tipos de explotaciones de diferente dimensión:

- Minifundios. Son aquellas explotaciones con un tamaño menor a 15 hectáreas. En ellas, el trabajo familiar, en la mayoría de los casos, tiene un papel muy importante en la realización de las tareas que requiere el cultivo.

- Explotaciones intermedias. Son explotaciones con un tamaño comprendido entre las 15 y las 100 hectáreas. En ellas, la producción se organiza sobre la base de la participación tanto del trabajo familiar como del asalariado.

- Latifundios. Son aquellas explotaciones con un tamaño superior a las 100 hectáreas. Son explotaciones gestionadas, esencialmente, sobre la base de personal asalariado.

El estudio del cultivo del olivar se complementa incorporando sus rendimientos. En este rubro se distinguen cuatro sistemas productivos muy diferentes:

- Olivar de secano de rendimientos bajos. Es un cultivo que suele darse en suelos poco favorables para cualquier otro cultivo agrícola en general, y para el olivar en particular. En una campaña, su rendimiento medio suele ser inferior a los 1,000 kilogramos de aceitunas por hectárea.

- Olivar de secano de rendimientos medios. Constituye un sistema productivo que se encuentra en suelos que tradicionalmente venían siendo ocupados por el olivar, pero que no ofrecen rendimientos especial mente el evados. Tiene rendimientos medios de entre 1,000 a 2,500 kilogramos de aceituna/hectárea anuales.

- O livar de secano de rendimientos altos. Es una modalidad de cultivo establecida en suelos especialmente adaptados al cultivo olivarero, con rendimientos de entre 2,500 a 4,000 kilogramos de aceituna/hectárea anuales.

- O livar de regadío. Es un cultivo que se localiza en suelos con características que en buena medida favorecen el desarrollo del olivar y disponen de un sistema de riego que supone que por término medio supere los 4,000 kilogramos de aceitunas/ hectárea anuales. 
El cruce de ambos criterios supone la diferenciación de 12 categorías, tal y como se expone en el cuadro $5 .{ }^{7}$ Así, por ejemplo, en el caso del olivar de secano de bajos rendimientos se distinguen minifundios, explotaciones intermedias y latifundios. Además, se cuantifica la importancia de cada una de las 12 categorías de acuerdo con tres variables: la participación en el número total de explotaciones, en la superficie total cultivada y en la producción total.

Cada una de ellas tiene un sentido distinto. La participación en el número de explotaciones indica la importancia social de cada una de las modalidades. Del mismo modo, la participación en la superficie puede considerarse un indicador de la importancia ecológica de cada modalidad de cultivo. Por último, la participación en la producción puede considerase un indicador de la importancia económica de cada tipo de explotación.

Del análisis del cuadro 5 se desprenden una serie de conclusiones. Por ejemplo, que los minifundios suponen más de $94 \%$ de las explotaciones. Esta importancia, como puede observarse, se distribuye casi por igual entre los cuatro tipos de minifundios. Sin embargo, en términos económicos existen fuertes diferencias entre los distintos tipos de minifundios. Así, mientras los minifundios de secano de bajos rendimientos representan tan sólo 3.6\% de la producción, los minifundios de regadío suponen $30.0 \%$. El resto se reparte entre las dos modalidades restantes (minifundios de secano de rendimientos al tos y medios), que representan, respectivamente, 10.9 y $11.9 \%$ de la producción total.

Por tanto, el resultado de la lógica productivista que ha dominado la evolución del olivar en los últimos decenios ha sido la concentración de la producción en explotaciones muy específicas con un rendimiento muy superior al resto. Entre ellas destaca el minifundio de regadío, que supone casi una tercera parte de la producción andaluza.

Además, se observa la misma diferencia en las explotaciones intermedias, aunque de una forma no tan acusada. La participación en la producción total de las explotaciones intermedias de

\footnotetext{
${ }^{7}$ Para calcular la importancia en la producción de cada tipo de olivar, se ha consultado a agricultores y expertos, y se concluyó que todas las explotaciones del olivar de secano de bajos rendimientos tienen los mismos rendimientos medios (kilogramos de aceituna/hectárea) con independencia de su tamaño. En una situación similar se encuentran las explotaciones del olivar de secano de rendimientos medios. Por el contrario, los rendimientos medios de los minifundios del olivar de secano de rendimientos altos superan en $10 \%$ a los de las explotaciones intermedias, y los de éstas a su vez, a los de los latifundios. Esta situación se repite en el caso del olivar de regadío.
} 


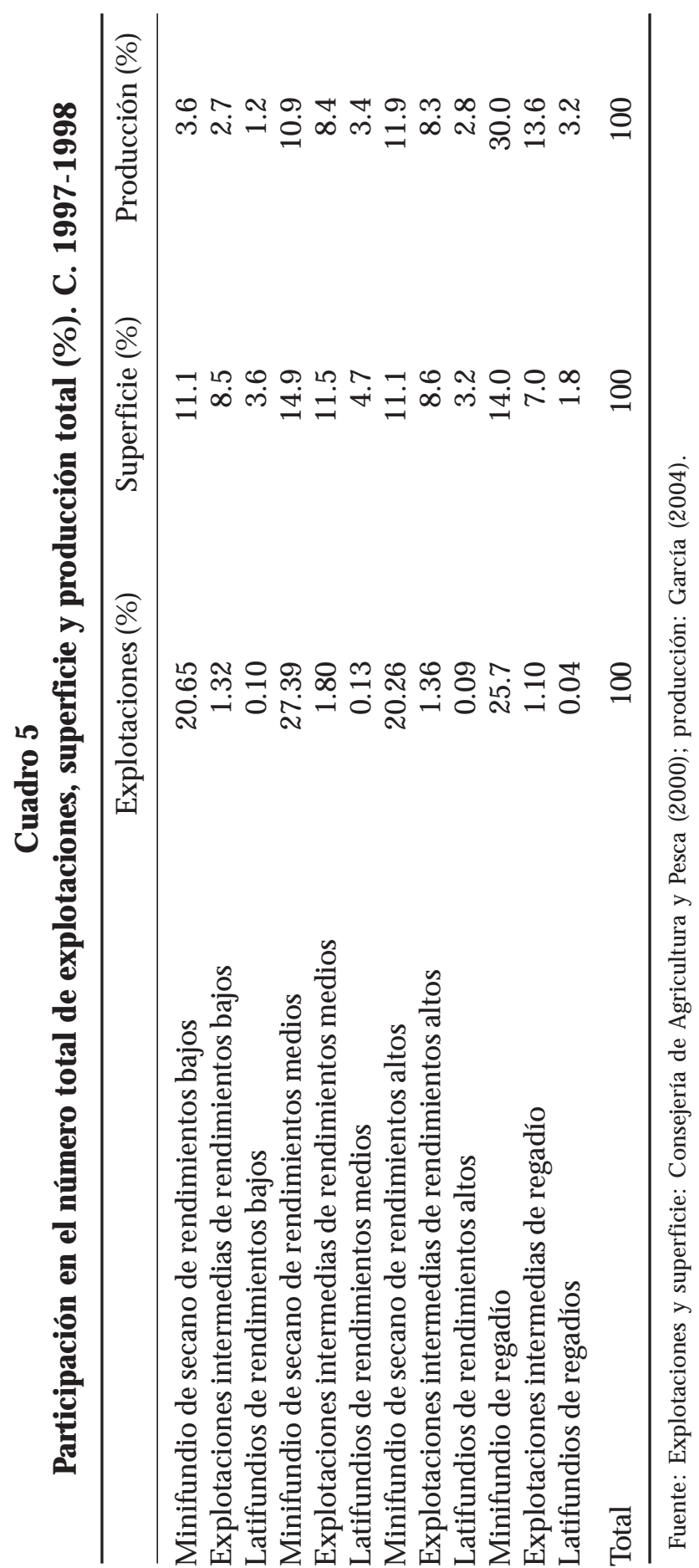


regadío, de rendimientos altos y de rendimientos medios es parecida: $13.6,8.3$ y $8.4 \%$, respectivamente. A las del olivar de secano de rendimientos bajos les corresponde, por el contrario, $2.7 \%$.

La situación en los latifundios es distinta, porque sólo uno de cada 10 latifundios funciona bajo regadío. Esto explica por qué la participación en la producción total de los latifundios de regadío es relativamente reducida (3.2\%).

Con la finalidad de estudiar la importancia del trabajo familiar y su relación con el tamaño de las explotaciones y los rendimientos del cultivo, se realizaron varias entrevistas semiestructuradas. La selección final de los propietarios de las explotaciones a los que se les realizaría la entrevista tenía cierto grado de dificultad. El olivar es un cultivo que presenta múltiples peculiaridades sobre la base de criterios tan dispares como el tamaño de las explotaciones, la productividad o las variedades de los olivos empleadas. Además, no se dispone de una base nominativa que permita acceder a las distintas explotaciones de olivar existentes en Andalucía. N o obstante, era importante para el análisis captar la heterogeneidad que presenta el olivar andaluz.

Para ello se seleccionaron cuatro municipios que reflejan en buena medida la diversidad existente en el olivar andaluz. En concreto, éstos fueron: Pozoblanco (Córdoba), El Saucejo (Sevilla), La Puebla de Cazalla (Sevilla) y Úbeda (Jaén). Pozoblanco es un área de olivares de secano de rendimientos bajos; El Saucejo, de olivares de secano de rendimientos medios; en La Puebla de Cazalla predominan los olivares de secano de rendimientos altos, y en Úbeda, los olivares de regadío. ${ }^{8}$ Fueron técnicos y gerentes de las cooperativas existentes en los municipios quienes proporcionaron los contactos con los propietarios a los que se les realizarían las entrevistas. En el cuadro 6 se muestra la distribución de las 83 entrevistas que se han realizado (véase el cuadro 6).

A cada uno de los entrevistados se le preguntaba personalmente por el número de jornales (días de trabajo) que correspondían al trabajo asalariado y al familiar. Además, se requería que distinguiera dentro del trabajo familiar, las aportaciones del agricultor, la esposa y los restantes miembros de la familia. Por

${ }^{8}$ La selección se realizó a partir de una base de datos municipal de Andalucía en la que figuran los rendimientos medios para la campaña 1997-1998 (Consejería de Agricultura y Pesca, 2000). El hecho de que este trabajo de investigación comprenda un periodo de dos años (1997 y 1998) se debe a que el ciclo productivo del olivar se extiende desde el 1 de noviembre de 1997 hasta el 31 de octubre de 1998. 


\section{Entrevistas realizadas. Campaña 1997-1998}

\begin{tabular}{lcccc}
\hline & M inifundios $\begin{array}{c}\text { Explotaciones } \\
\text { intermedias }\end{array}$ & Latifundios Total \\
\hline $\begin{array}{l}\text { Olivar de secano } \\
\text { de rendimientos bajos }\end{array}$ & 10 & 7 & 3 & 20 \\
$\begin{array}{l}\text { Olivar de secano } \\
\text { de rendimientos medios }\end{array}$ & 10 & 7 & 4 & 21 \\
$\begin{array}{l}\text { Olivar de secano } \\
\text { de rendimientos altos }\end{array}$ & 10 & 7 & 4 & 21 \\
$\begin{array}{l}\text { Olivar de regadío } \\
\text { Total }\end{array}$ & 10 & 7 & 4 & 21 \\
\hline
\end{tabular}

Fuente: García (2004).

último, se preguntó sobre la importancia de los ingresos obtenidos por los propietarios en actividades ajenas al olivar, y el tiempo de dedicación a ellas.

\section{La participación del trabajo familiar en las explotaciones del olivar}

La participación del trabajo familiar en el olivar andaluz no es, en ningún caso, despreciable. En el cuadro 7 se advierte que el trabajo familiar representa casi $46 \%$ del trabajo total necesario en este cultivo. Es decir, básicamente la mitad del trabajo que genera el cultivo del olivar es atendido con la participación de los propios miembros de la explotación.

Además, se aprecia que la participación del trabajo familiar en el trabajo total es muy distinta de acuerdo con el tamaño de la explotación. Así, es mayoritaria en el caso del minifundio, donde el trabajo familiar representa más de la mitad del trabajo que requiere la explotación (52.8\%). Los latifundios se encuentran en el extremo contrario, pues allí el trabajo familiar es poco representativo (9.6\%). Entre ambos casos se encuentran las explotaciones intermedias, donde el trabajo familiar representa una cuarta parte del trabajo que necesita la explotación.

Si se contabiliza exclusivamente el trabajo familiar, puede verse que es generado casi exclusivamente por los minifundios. Éstos absorben $88.4 \%$ del trabajo familiar total, mientras que los lati- 


\section{C uadro 7}

Participación del trabajo familiar (\% ). C. 1997-1998

\begin{tabular}{lccc}
\hline $\begin{array}{c}\text { Tipo } \\
\text { de explotación }\end{array}$ & $\begin{array}{c}\text { Trabajo familiar } \\
\text { en el trabajo total } \\
\text { del segmento }\end{array}$ & $\begin{array}{c}\text { Trabajo familiar } \\
\text { del segmento } \\
\text { sobre el trabajo } \\
\text { familiar total }\end{array}$ & $\begin{array}{c}\text { Trabajo familiar } \\
\text { del segmento } \\
\text { sobre el trabajo } \\
\text { total }\end{array}$ \\
\hline M inifundio & 52.8 & 88.4 & 40.1 \\
Explot. interm. & 24.4 & 10.9 & 4.9 \\
Latifundios & 9.6 & 0.7 & 0.4 \\
\hline
\end{tabular}

Fuente: García (2004).

fundios, por el contrario, sólo generan $0.7 \%$. Así, los minifundios, que representan $94 \%$ de las explotaciones y $56.4 \%$ de la producción, participan con casi $90 \%$ del trabajo familiar. Es decir, los minifundios no sólo son relevantes por el importante número de pequeños propietarios y por su alta participación en la producción total, sino también porque fundamentalmente constituyen una destacada fuente de trabajo familiar.

\subsection{La distribución del trabajo familiar en el olivar andaluz. EI papel del trabajo de la mujer}

El análisis de la relación entre el trabajo familiar y el tipo de explotación se completa con el estudio de la distribución del trabajo entre los distintos miembros de la familia. Lo primero que debe afirmarse es que el trabajo del cabeza familia supone casi $60 \%$ del trabajo familiar total, y se completa con las aportaciones del resto de los miembros de la familia (fundamentalmente la mujer y los hijos). Así, la importancia del trabajo familiar en las explotaciones se debe en buena medida a la participación del cabeza de familia en las tareas que necesita el cultivo del olivar.

Existe, por tanto, un importante y creciente desarraigo de los restantes miembros de la explotación. Esto supone un debilitamiento de las estructuras patriarcales, en la medida que los intereses de la familia ya no se subordinan a los de la explotación, sino que se vinculan a actividades ajenas.

Este fenómeno es calificado en algunos trabajos que toman como base la agricultura española, como desfamiliarización de la explotación. 
Es decir, que la explotación agraria familiar es cada vez menos familiar, en la medida que la familia, como conjunto, progresivamente participa menos en las decisiones que afectan a la explotación y también participa menos en el trabajo de la misma, a la vez que sus miembros orientan sus estrategias educativas, laborales o matrimoniales al margen de aquélla y de la agricultura (González y Gómez, 2002: 429).

El hecho de que el trabajo familiar represente en los minifundios casi $53 \%$ del trabajo total, hace necesario que analicemos la participación del cabeza de familia y de los restantes miembros de la familia en este tipo de explotaciones. En el cuadro 8 se advierte que el trabajo del cabeza de familia tiene un papel muy destacado en el trabajo familiar de todos los tipos de minifundios, y principalmente en el caso del olivar de secano de rendimientos altos, en el que alcanza $84 \%$ del trabajo familiar total. El trabajo de los restantes miembros de la familia, por el contrario, tiene un papel secundario y complementa la aportación del cabeza de familia. Tan sólo en el caso del olivar de secano de rendimientos medios es la base fundamental al representar $57 \%$ del trabajo familiar total.

Así, en la actualidad se advierte una importante desfamiliarización de las explotaciones del olivar en Andalucía, pues la mayoría de las veces la aportación de los miembros de la familia distintos del cabeza de familia no es la base del trabajo familiar de este cultivo. Es decir, existe por parte de los hijos y la esposa

\section{C uadro 8}

Distribución del trabajo familiar. C. 1997-1998

\begin{tabular}{lcc}
\hline Segmento & $\begin{array}{c}\text { \% de trabajo } \\
\text { del cabeza de familia } \\
\text { en el trabajo familiar } \\
\text { del segmento }\end{array}$ & $\begin{array}{c}\text { \% de trabajo } \\
\text { de otros miembros } \\
\text { de la familia en } \\
\text { el trabajo familiar } \\
\text { del segmento }\end{array}$ \\
\hline $\begin{array}{l}\text { M inifundio de secano } \\
\text { de rend. bajos }\end{array}$ & 58.6 & 41.4 \\
$\begin{array}{l}\text { M inifundio de secano } \\
\text { de rend. medios }\end{array}$ & 43.0 & 57.0 \\
$\begin{array}{l}\text { M inifundio de secano } \\
\text { de rend. altos }\end{array}$ & 84.0 & 16.0 \\
M inifundio de regadío & 59.5 & 40.5 \\
\hline
\end{tabular}

Fuente: García (2004). 
del agricultor una escasa implicación en las tareas que requiere la explotación.

Esta ruptura entre la explotación y la familia tiene sus orígenes en la reestructuración que siguió el cultivo en la década de los setenta, y los procesos de globalización económica lo han acentuado. Así lo anticipaba Agustín López (1980: 34): “H oy la familia está reducida al mínimo, prácticamente queda marido y mujer. No sólo han emigrado obreros sino muchos hijos de pequeños y medianos agricultores. Uno a la Telefónica, el otro a la Renfe (Red Oficial de Ferrocarriles Españoles), otro a la Guardia Civil y, acaso llegó para que uno se hiciese maestro".

Una de las posibles explicaciones de gran parte de la pérdida del carácter familiar de las explotaciones del olivar en Andalucía se encuentra en la escasa rentabilidad económica que presenta este cultivo. Así, la renta media anual que obtiene una explotación es de 5,579.4 euros (García, 2004). Esta reducida renta no garantiza los ingresos suficientes para que las explotaciones puedan satisfacer todas necesidades, ni alcanzar una estabilidad económica que sirva como atractivo para que los hijos de los propietarios agrarios permanezcan en la actividad. En este mismo sentido, en un trabajo sobre el caso particular de M éxico se sostiene que como consecuencia de la dinámica capitalista, los pequeños agricultores deben completar sus ingresos con los provenientes en otras actividades. La parcela ya no es el domicilio de la familia; sus integrantes emigran buscando alternativas de subsistencia, y a menudo deben aportar al sostenimiento de quienes se quedan, normalmente trabajadores en el ocaso de la vida (Figueroa, 2005).

Además, el proceso de modernización que ha seguido el olivar en los últimos años ha supuesto la incorporación de nuevas maquinarias (vibradores) que reducen las necesidades de mamo de obra. La mecanización de la recolección de la aceituna, que es la tarea del olivar que más mano de obra necesita, ha supuesto pérdidas anuales de más de cuatro millones de jornales (García, 2004), Io que ha aumentado la temporalidad del mercado de trabajo en el cultivo del olivar y perjudicado seriamente la estabilidad laboral de todos los miembros de la explotación.

A ello se unen las estrategias de los propios agricultores en favor de la formación de sus hijos, en perjuicio del patrimonio familiar. Se invierte la mayor parte del excedente familiar en "dar una carrera" a los hijos (González y Gómez, 1997). Esto supone un distanciamiento de la explotación por parte de los hijos y pone 
en peligro el relevo generacional, pues participan en muy poca medida en la dinámica de acumulación de la explotación.

Un aspecto muy interesante en la participación del trabajo familiar en la explotación lo constituye la aportación de la mujer. En general, esa aportación en el cultivo del olivar representa $13.1 \%$ del trabajo familiar total.

De nuevo se estudia el caso del minifundio, pues es el que absorbe la mayor parte del trabajo de las mujeres, con una participación de $12.9 \%$ del trabajo familiar total. En el cuadro 9 se advierte que el trabajo de la mujer, en la mayoría de las ocasiones, complementa la aportación del cabeza de familia y no constituye, en ningún caso, una fuente principal de rentas. Únicamente en el caso de los minifundios menos productivos, la participación femenina en el trabajo familiar es relativamente importante. En los restantes casos, es muy reducida.

Esta escasa participación de la esposa del agricultor en las tareas que requiere el cultivo del olivar puede explicarse por la estructura patriarcal de la organización familiar de la explotación, en la que al hombre se le asigna el trabajo agrícola y a la mujer el trabajo doméstico. De este modo, el hombre realiza básicamente las tareas que requiere el olivar, y la mujer sólo participa en determinados periodos en los que se requiere una abundante mano de obra, como es el caso de la recolección de la aceituna. Esta situación supone que la mujer se encuentra en una posición muy subordinada a los intereses de la explotación y en

C uadro 9

Trabajo de la mujer. C . 1997-1998

\begin{tabular}{lcc}
\hline Segmento & $\begin{array}{c}\text { \% de trabajo } \\
\text { de la mujer en } \\
\text { el trabajo familiar } \\
\text { del segmento }\end{array}$ & $\begin{array}{c}\text { \% de trabajo } \\
\text { de la mujer en } \\
\text { el trabajo total } \\
\text { del segmento }\end{array}$ \\
\hline $\begin{array}{l}\text { M inifundio de secano } \\
\text { de rend. bajos }\end{array}$ & 28.8 & 14.2 \\
$\begin{array}{l}\text { M inifundio de secano } \\
\text { de rend. medios }\end{array}$ & 6.1 & 4.5 \\
$\begin{array}{l}\text { M inifundio de secano } \\
\text { de rend. altos }\end{array}$ & 12.9 & 4.6 \\
M inifundio de regadío & 15.6 & 8.9 \\
\hline
\end{tabular}

Fuente: García (2004). 
la que su trabajo es invisible y con escaso reconocimiento social. El trabajo de la mujer en buena medida complementa al del agricultor y carece de autonomía.

N o obstante, en los últimos años las mujeres están desarroIlando actividades fuera de la explotación, con lo que logran mayor independencia al salir de la unidad familiar y trabajar en otras áreas. Así lo señaló la mujer de un agricultor:

\begin{abstract}
M i situación es muy distinta de la que vivieron mis padres. M i padre hacía y deshacía lo que quería en la explotación, y mi madre siempre estaba trabajando en la casa y tenía poca participación en las decisiones que se tomaban. Sin embargo, hoy, aunque mi marido trabaja en la explotación y yo como profesora en un colegio, él me consulta todo y asisto como propietaria a las reuniones que se celebran en la cooperativa que afectan a nuestra explotación.
\end{abstract}

Esta importante transformación en el papel de la mujer en la explotación puede explicarse por la masiva incorporación de las mujeres a los estudios superiores y, en concreto, a los universitarios. En el caso de M éxico se advierte que a partir de los años setenta se ha incrementado de un modo muy importante la participación de la población femenina en los estudios superiores, que incluso supera a la de los hombres. Esto ha generado una también mayor participación de la mujer en la actividad económica, lo que a su vez les permite una mayor autonomía y una posición más favorable para negociar tanto en el ámbito familiar como en el laboral (Luna, 2005).

\title{
4.2. La reproducción de las explotaciones familiares
}

En la sección anterior se ha puesto de manifiesto que la principal aportación del trabajo familiar se debe al cabeza de familia. Un elemento de especial importancia asociado con el trabajo familiar lo constituyen las actividades ajenas al olivar. Podemos conocer la incidencia económica que tienen los ingresos obtenidos en actividades diferentes de la propia explotación y prever con ello el futuro de las explotaciones familiares.

La pluriactividad es un rasgo frecuente en el sector agrario. En Andalucía cerca de $20 \%$ de los titulares de las explotaciones y sus cónyuges, y entre 20 y $25 \%$ en Asturias y Cataluña, la practican (Etxezarreta et al., 1995). En un trabajo sobre las explotaciones familiares en Andalucía, Josefina Cruz y otros autores (1980: 48) expresaban: "Todos los tipos de componentes de la 
fuerza de trabajo familiar se emplean fuera de la explotación propia; casi el $50 \%$ de los empresarios o titulares de las explotaciones, el $80 \%$ de los hijos y prácticamente la totalidad del resto de los activos familiares (esposas e hijas principalmente)".

En el olivar andaluz, la situación no es distinta. Prueba de ello es que en 1989 sólo $1.2 \%$ de los titulares trabajaban a tiempo completo y $46.2 \%$ tenían otra actividad lucrativa, que en $84 \%$ de los casos era su actividad principal (INE, 1989). El transcurso del tiempo ha acentuado el trabajo de los propietarios en otras actividades distintas de las del olivar. No obstante, se advierte que la pluriactividad no tiene la misma importancia en todos los tipos de olivar. ${ }^{9}$ En el cuadro $10^{10}$ se aprecia cómo en todos los minifundios, a excepción del olivar de regadío, el trabajo en actividades ajenas a la propia explotación tiene un peso importante tanto en tiempo de trabajo como en ingresos. Para las explotaciones intermedias, únicamente en el caso del olivar de secano de rendimientos bajos la pluriactividad alcanza una cierta importancia. O tro tanto ocurre en el caso de los latifundios.

Esta situación convierte al cultivo del olivar más que en la actividad principal, en una actividad complementaria para las explotaciones. Cabe destacar el hecho de que son los minifundios de regadío los únicos minifundios en los que las actividades diferentes al olivar tienen una importancia reducida. Así, to do parece indicar que la intensificación de la producción ha impedido a los pequeños propietarios (minifundios de regadío) su exclusión del mercado, de forma que todavía es posible el mantenimiento de una familia a partir de la renta derivada de los minifundios.

Sobre la base de la importancia del tiempo dedicado a las actividades ajenas al olivar y los ingresos de allí derivados, así como de la participación de cada una de las categorías en el número total de explotaciones y producción total, podemos distinguir los siguientes tipos de explotaciones:

- Un olivar que tiende al abandono, y sin relevo generacional. El olivar de secano de rendimientos bajos, los minifundios

${ }^{9}$ El Censo Agrario de 1999 elaborado por el INE no comprende la información referente a la participación de las explotaciones de olivar en otras actividades diferentes. Esto no nos permite comparar la situación de 1989 y 1999.

10 En ese cuadro excluyo a los agricultores que están jubilados, pues además de haber terminado su vida laboral, en ningún caso realizan trabajos en actividades distintas a la de su propia explotación. Se han considerado todos los ingresos y la dedicación a las actividades ajenas al olivar, los procedentes de otros cultivos propios de la explotación, y las prestaciones sociales recibidas. 


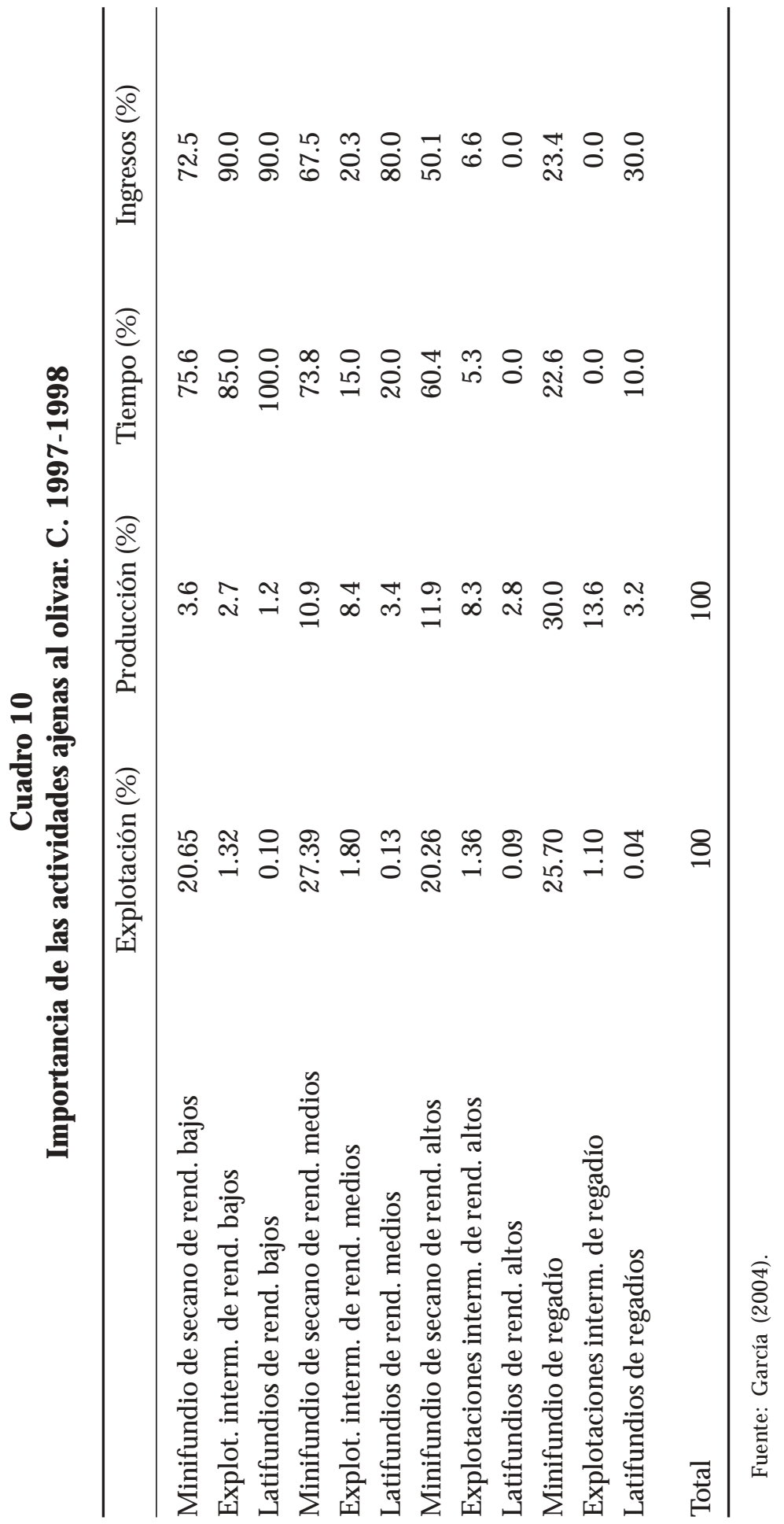


del olivar de rendimientos medios, e incluso los minifundios del olivar de rendimientos altos están muy posiblemente condenados al abandono. Ello afecta a más de $69.7 \%$ de las explotaciones, a $49.2 \%$ de la superficie y a más de $30 \%$ de la producción. Dadas las condiciones actuales, la renovación generacional en este tipo de explotaciones resulta muy difícil y, por tanto, su supervivencia a medio plazo se ve amenazada.

- Un olivar que sobrevive de la autoexplotación de sus propietarios y los demás miembros de la familia. En este grupo se incluirían los minifundios del olivar de regadío, que represen$\tan 25.7 \%$ de las explotaciones y $30 \%$ de la producción; las explotaciones intermedias del olivar de secano de rendimientos medios, y las de secano de rendimientos altos. En los tres casos se generan rentas suficientes como para mantener una unidad familiar, aunque ello sea sobre la base de la utilización intensiva del trabajo familiar.

- Un olivar que acumula importantes niveles de rentas de capital. Las explotaciones intermedias de regadío y todos los tipos de latifundios, con excepción del secano de rendimientos bajos, tienen todavía la suficiente capacidad de generación de rentas como para permitir la existencia de procesos de acumulación de capital en su interior.

En cuanto al tipo de actividades que se compaginan con el olivar, se advierte que los propietarios de minifundios comparten mayoritariamente el trabajo en su explotación con ocupaciones en otras explotaciones de olivar como mecanismo de subsistencia. De este modo, ser propietario de una pequeña explotación se convierte en un medio que facilita relaciones de trabajo en explotaciones intermedias y latifundios. Esto, además, hace que el minifundio sea considerado como un refugio al que se puede volver cuando finalizan las tareas en otras explotaciones.

Los grandes propietarios agrícolas, por el contrario, trabajan en actividades distintas del cultivo del olivar, en sectores como la industria agroalimentaria, la enseñanza, la construcción, la sanidad, la ganadería y otros cultivos agrícolas. Esto se asocia con la existencia en este cultivo de propietarios absentistas, que reciben una renta por el mero hecho de ser dueños de la tierra, sin participar activamente en las tareas que requiere este cultivo. 


\section{Conclusiones}

A lo largo de la historia, el cultivo del olivar ha tenido un destacado papel en la estructura económica y social de Andalucía. En los años sesenta y setenta, las instituciones públicas españolas impulsaron que los propietarios agrícolas españoles, y en particular los andaluces, aplicaran los principios de la revolución verde (agroquímicos, maquinarias, plaguicidas, regadío, selección de variedades, etc.). Este proceso de reestructuración económica aumentó los niveles de producción y propició la desaparición del olivar tradicional y el carácter ecológico que presentaba este sistema productivo.

Además, originó la ruptura entre la explotación y la familia, pues la mayoría de los miembros de la familia abandonaron la explotación y comenzaron a desarrollar otras actividades distintas. Esto supuso que la participación del trabajo familiar en la explotación sufriera una importante reducción.

En este trabajo se muestra cómo la aplicación de la Política Agraria Comunitaria al cultivo del olivar andaluz en las décadas de los ochenta y los noventa ha aumentado de nuevo la producción del olivar, los costes ambientales y el desarraigo de los miembros de la familia distintos al cabeza de familia, pues los hijos buscan su modo de vida en actividades distintas a la del cultivo del olivar.

En el caso de la mujer se advierte que se ha producido una incorporación a actividades ajenas a este cultivo y una participación más activa en la gestión de la explotación, lo que ha favorecido su autonomía, su reconocimiento social y su posición en la explotación.

A todo ello se une el hecho de que buena parte de las explotaciones pequeñas y medianas presentan condiciones económicas que dificultan su reproducción y permanencia. En ellas, el relevo generacional resulta gravemente difícil. Es decir, no existen condiciones económicas favorables para que los hijos de los propietarios se dediquen al cultivo del olivar, a tiempo completo y como actividad principal. Además, se advierte que esta situación se repite en M éxico, donde la explotación ha dejado de ser el sustento de la familia y se tiene que completar los ingresos con los obtenidos en otras actividades, además de que los hijos buscan nuevos modos de vida.

Así, es necesario que se produzcan cambios en el sistema de subvenciones de la Política Agraria Comunitaria en relación con el 
olivar, que favorezcan el olivar ecológico y el relevo generacional, en particular en aquellos espacios con mayores problemas de desarrollo económico. Además, los productores agrarios tienen que desarrollar fórmulas de economía social (cooperativas) para garantizar su permanencia en un entorno cada vez más competitivo.

\section{Bibliografía}

Abad, Carlos y José M anuel N aredo (1997), "Sobre la modernización de la agricultura española (1940-1995): de la agricultura tradicional a la capitalización agraria y dependencia asistencial", Agricultura y sociedad en la España contemporánea, Centro de Investigaciones SociológicasM inisterio de Agricultura, Pesca y Alimentación, M adrid, pp. 249-316.

Angles, Stephane (1999), “Evolución de la geografía oleícola en Andalucía", revista Olivae, Consejo O leícola Internacional, M adrid, 78: 12-22.

Bernal, Antonio M iguel (1979), “Cambio, modernización y problemas en la agricultura andaluza (SS. xIx-xx)", Revista de Estudios Regionales, Universidades de Andalucía, M álaga, 1: 113-129.

Cano Orellana, Antonio (2000), "El olivar al servicio del proyecto 'modernizador'. Crisis de un modelo de cultivo tradicional", Desde el Sur, M ergablum, Sevilla, 3: 1-37.

Civantos, Luis (1997), "La olivicultura en el mundo y en España”, El cultivo del olivo, M undi-Prensa-Consejería de Agricultura y Pesca, Junta de Andalucía, M adrid, pp. 17-35.

Consejería de Agricultura y Pesca (2000), Superficie y producción del olivar en Andalucía, Junta de Andalucía, Sevilla (inédito).

(2005), Plan andaluz de la agricultura ecológica, Junta de Andalucía, Sevilla. Disponible en: http:// www.juntadeandalucia.es/agriculturaypesca/prospectiva/ Ecologico1_doc.pdf, 31 de octubre de 2005. 
Consejo Oleícola Internacional (2002), “El mercado mundial del aceite de oliva", revista Olivae, Consejo Oleícola Internacional, M adrid, 92: 22-26.

Cruz Villalón, Josefina, Juan Francisco Ojeda Rivera y Florencio Zoido N aranjo (1980), “Explotación familiar y estrategias campesinas en los regadíos béticos", revista Agricultura y Sociedad, M inisterio de Agricultura, Pesca y Alimentación, M adrid, 17: 11-67.

Delgado Cabeza, M anuel y Andrés Vázquez Duarte (2002), "M odernización y crisis de la agricultura en Andalucía. 1955-1995", La historia de Andalucía a debate, II. El campo andaluz, Anthropos, Granada, pp. 179-206.

D onaire, Gines (2005), “La superficie de olivar de regadío se ha multiplicado por cuatro desde 1980", El País, 20 de junio.

Etxezarreta Zubizarreta, M iren, Josefina Cruz Villalon, M ario García M orilla y Lourdes Viladomiu Canela (1995), La agricultura familiar, ante las nuevas políticas agrarias comunitarias, M inisterio de Agricultura, Pesca y Alimentación, Secretaría General Técnica, Serie Estudios, M adrid.

Fernández Latorre, Alfredo (1927), El cultivo del olivo en la provincia de Sevilla, Savats Editores, Sevilla.

Figueroa, Víctor M anuel (2005), “América Latina: descomposición y persistencia de lo campesino", revista Problemas del Desarrollo, M éxico, 36 (142). Disponible en: http:// probdes.iiec.unam.mx/interiores/numero142.htm, 28 de octubre de 2005.

García Brenes, M anuel David (2004), La reestructuración de la cadena de valor del aceite de oliva en Andalucía. Impactos ecológicos, sociales y económicos, tesis doctoral, Sevilla.

García, Carlos, Pedro Pérez y Francisco Fuentes (1997), “Economía del aceite de oliva", El cultivo del olivo, M undiPrensa-C onsejería de Agricultura y Pesca, Junta de Andalucía, M adrid, pp. 615-651. 
Gerritsen, Peter R. W., M aría M ontero y Pedro Figueroa (2003), "EI mundo en un espejo. Percepciones campesinas de los cambios ambientales en el O ccidente de M éxico", revista Economía, Sociedad y Territorio, Iv (14): 253-278. Disponible en: http://www.cmq.edu.mx/revista_EST.htm, 27 de octubre de 2005.

González Rodríguez, J uan J esús y Cristóbal Gómez Benito (1997), "Clases agrarias, estrategias familiares y mercado del trabajo", Agricultura y sociedad en la España contemporánea, Centro de Investigaciones Sociológicas-M inisterio de Agricultura, Pesca y Alimentación, M adrid, pp. 565-580.

(2002), "Una nota sobre la evolución del mercado de trabajo agrario", Agricultura y sociedad en el cambio de siglo, Centro de Investigaciones Sociológicas-M inisterio de Agricultura, Pesca y Alimentación, M adrid, pp. 451-458.

Guerrero G arcía, Andrés (1997), N ueva olivicultura, M undi-Prensa, M adrid.

H umanes Guillén, J osé (1996), "El olivar español en el siglo xx।", revista Agricultura, suplemento, M inisterio de Agricultura, Pesca y Alimentación, M adrid, pp. 910-915.

Ibáñez, Jesús (2002), "Perspectivas de la investigación social: el diseño en las tres perspectivas", El análisis de la realidad social. Métodos y técnicas de investigación, Alianza Editorial, M adrid, pp. 57-98.

INE (Instituto N acional de Estadística) (1972), Censo agrario. Re sultados por comunidades autónomas, Andalucía, INE, $M$ adrid.

(1989), Censo agrario. Resultados por comunidades autónomas, según la orientación técnico económica, INE, M adrid.

López O ntiveros, Agustín (1980), ¿Q ué pasa con el olivar?, Publicaciones del Instituto de Desarrollo Regional, Granada. 
Luna Santos, Silvia (2005), "Avances en educación superior. Irrupción femenina y continuidad masculina", revista Economía, Sociedad y Territorio v, (17): 219-246. Disponible en: http://www.cmq.edu.mx/revista_EST.htm, 26 de octubre de 2005.

M alefakis, Eduard (2001), Reforma agraria y revolución campesina en la España del siglo xx, Austral, Barcelona.

MAPA (M inisterio de Agricultura, Pesca y Alimentación) (1988), El olivar español. Planes de reestructuración y reconversión, Dirección General de Producción Agraria, M adrid.

(2001), Encuesta sobre superficies y producción, MAPA, M adrid.

N aredo Pérez, José M anuel (1983), "La crisis del olivar como cultivo 'biológico' tradicional", revista Agricultura y Sociedad, M inisterio de Agricultura, Pesca y Alimentación, M adrid, 26: 168-288.

(1996), La evolución de la agricultura en España (19401990), G ráfica N ueva, Granada.

O rtega Alba, Francisco (1975), “El olivar. Algunos problemas de base y crisis en Andalucía Oriental y Córdoba", Revista del Instituto de Comercio Exterior, $\mathrm{M}$ inisterio de Industria, Comercio y Turismo, M adrid, pp. 69-85.

Parra, Fernando (1988), "La dehesa y el olivar", Enciclopedia de la naturaleza en España, D ebate, M adrid, pp. 77-112.

Sevilla Guzmán, Eduardo y M anuel González de M olina N avarro (1990), "Ecosociología: algunos elementos teóricos para el análisis de la coevolución social y ecológica en la agricultura", Revista Española de Investigaciones Sociológicas, M adrid, pp. 7-45.

Recibido: 24 de agosto de 2005 Reenviado: 3 de noviembre de 2005. Aceptado: 9 de diciembre de 2005. 
M anuel David García Brenes es doctor en ciencias económicas y empresariales por la Universidad de Sevilla (2004), profesor ayudante del D epartamento de Economía Aplicada ॥ en la Escuela Universitaria Ingenieros Técnicos Agrícolas, y miembro del grupo de investigación AREA (Análisis Regional de Economía Andaluza) y de la red internacional SYAL (Systémes agroalimentaires localisés). Es autor de varios artículos en revistas de divulgación científica y coautor de libros en temas de agricultura, industria agroalimentaria, cooperativismo, etc., desde las perspectivas social, ecológica y económica; por ejemplo: “Los grandes grupos alimentarios y las cooperativas en el sector del aceite de oliva en Andalucía", Cooperativismo agrario y desarrollo rural, Universidad Politécnica de Valencia, pp. 527-544, 2005; "Producción y comercialización del aceite de oliva en Andalucía", Boletín Económico de Andalucía, 31-32: 197-207, Secretaría de Economía y H acienda, Junta de Andalucía, 2002, y "Una aproximación a las externalidades de la agricultura andaluza", El desarrollo rural en la agenda 2000, 142: 520-537, M inisterio de Agricultura, Pesca y Alimentación, 1999. 